Revue de droit comparé du travail et de la sécurité sociale

1 | 2020

Doctrine

\title{
La protection du droit syndical en Turquie - le droit et les réalités
}

Melda Sur

\section{(2) OpenEdition}

12 Journals

Édition électronique

URL : https://journals.openedition.org/rdctss/1169

DOI : $10.4000 /$ rdctss. 1169

ISSN : 2262-9815

Éditeur

Centre de droit comparé du travail et de la sécurité sociale

Édition imprimée

Date de publication : 1 avril 2020

Pagination : 22-33

ISSN : 2117-4350

Référence électronique

Melda Sur, «La protection du droit syndical en Turquie - le droit et les réalités », Revue de droit comparé du travail et de la sécurité sociale [En ligne], 1 | 2020, mis en ligne le 01 novembre 2021, consulté le 11 novembre 2021. URL : http://journals.openedition.org/rdctss/1169 ; DOI : https:// doi.org/10.4000/rdctss. 1169

\section{(c) (†) $९$}

Revue de droit comparé du travail et de la sécurité sociale est mise à disposition selon les termes de la Licence Creative Commons Attribution - Pas d'Utilisation Commerciale - Pas de Modification 4.0 International. 


\title{
LA PROTECTION DU DROIT SYNDICAL EN TURQUIE - LE DROIT ET LES RÉALITÉS
}

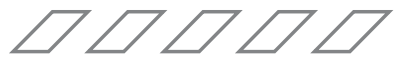

\section{RÉSUMÉ}

La Turquie est partie aux Conventions de I'OIT concernant les libertés syndicales (Conventions $\mathrm{n}^{\circ} 87$, 98, 151 et 135). Le droit syndical est constitutionnellement reconnu et réglementé par une législation plutôt élaborée, assortie de garanties contre les discriminations lors de l'embauche, pendant la durée de l'emploi et le licenciement. Toutefois, la mise en œuvre de ces garanties est aléatoire et se heurte aux difficultés de preuve et aux longueurs de la procédure judiciaire, aboutissant parfois à une sanction qui n'est pas assez dissuasive. Tenant compte de la conjoncture actuelle marquée par une crise économique et un taux élevé de chômage, de même qu'une inadéquation de certains syndicats à s'adapter aux réalités actuelles, la liberté syndicale et ses garanties juridiques se trouvent affaiblies devant les faits.

MOTS CLÉS: Liberté syndicale, entraves à la liberté syndicale, sanctions contre les discriminations, preuve de la discrimination, protection contre les licenciements, Turquie.

\begin{abstract}
Turkey is party to ILO Conventions no. 87, 98, 151 and 135 on the freedom of association. The freedom to organize and join trade unions is protected by the Constitution and a detailed set of guarantees has been provided by the legislation, with guarantees while recruiting, during employment and concerning termination of employment. However, the effective application of these guarantees meets obstacles, especially in the proof of the discrimination, and due to the length of judicial proceedings, infringements eventually result with a legal sanction that is far from being deterring. Within a situation of economic crisis and unemployment, and also due to inabilities of some trade unions to adapt themselves to new issues, it is difficult to say that in actual facts " all necessary and appropriate measures ensure that workers and employers exercise freely the right to organize ».
\end{abstract}

KEY WORDS: Right to organize, Freedom of Association, Sanctions against Anti Union Discriminations, Protection against Termination of Employment, Turkey. 
e droit syndical nécessite une protection particulièrement efficace en raison de son importance vitale dans les relations de travail et la définition des politiques sociales. La conception actuelle des droits syndicaux inclut non seulement le droit de fonder des syndicats et d'y adhérer, mais également le droit de négociation collective et le droit de grève, qui rendent son exercice plus efficace, mais suscitent aussi une certaine méfiance de la part de certains employeurs. II importe donc d'apporter des garanties suffisantes en vue de leur assurer un exercice effectif, de protéger efficacement le travailleur syndiqué contre des discriminations lors de l'embauche, pendant la durée de la relation de travail, et surtout contre les licenciements qui font l'objet de nombreux contentieux et ont donné lieu à une foisonnante jurisprudence.

Cette étude sur la protection du droit syndical en Turquie débutera ainsi par une présentation du système légal, avec une description des dispositions constitutionnelles et législatives (I). Ensuite seront analysées les difficultés quant à la mise en œuvre de ces protections légales, en particulier dans le domaine de la preuve, des longueurs procédurales et de la sanction prévue (II). L'étude tendra finalement à rechercher des solutions en vue de rendre plus effectif l'exercice du droit syndical dans un environnement dominé par des crises économiques et le chômage.

\section{I - LE DROIT SYNDICAL GARANTI PAR LA LÉGISLATION}

La liberté syndicale est un droit fondamental qui figure dans divers textes internationaux consacrés aux Droits de l'homme, et en particulier dans la Convention Européenne des Droits de l'Homme (art. 11), les Conventions n 87 et 98 de l'OIT et les deux Pactes des Nations Unies, le Pacte relatif aux droits civils et politiques (art. 22), ainsi que le Pacte relatif aux droits économiques, sociaux et culturels (art. 8); la Turquie étant partie à tous ces textes. La Turquie a également ratifié la Charte sociale européenne et la Charte révisée, en excluant toutefois les articles 5 et 6 consacrés aux droits syndicaux. Rappelons en outre qu'en 1998, la Déclaration de I'OIT relative aux principes et droits fondamentaux au travail a souligné le caractère de « droit fondamental » qui caractérise la liberté syndicale ${ }^{1}$. Ainsi, l'Organisation Internationale du Travail a instauré un système de suivi particulier, incluant même dans le mécanisme de contrôle les États qui n'ont pas ratifié les conventions fondamentales.

\section{A - L'ÉTENDUE DE LA GARANTIE CONSTITUTIONNELLE}

en droit turc, c'est depuis la Constitution de 1961 que les droits syndicaux (droit de se syndiquer, droit de négocier des conventions collectives et droit de grève) furent consacrés et élevés au rang de droits constitutionnels.

1 Au même titre que la liberté d'association et la reconnaissance effective du droit de négociation collective, l'élimination de toute forme de travail forcé ou obligatoire, l'abolition effective du travail des enfants, l'élimination de la discrimination en matière d'emploi et de profession. 
La Constitution actuelle de 1982 dispose en son article 51, intitulé « Droit de fonder des syndicats ", que "les travailleurs et employeurs ont le droit de fonder sans autorisation préalable des syndicats en vue de protéger et développer les droits et intérêts économiques et sociaux de leurs membres, d'y adhérer ou de les quitter librement. Nul ne peut être tenu d'adhérer ou de mettre fin à son adhésion à un syndicat » (alinéa 1 ${ }^{\mathrm{er}}$ ). "Le droit de fonder des syndicats ne peut être limité que par la loi et dans le but de sauvegarder la sécurité nationale, l'ordre public, la prévention du crime, la santé et moralité publiques et les droits et libertés d'autrui » (alinéa $2^{\mathrm{e}}$ ). "Les formes, conditions et procédures à suivre lors de la création des syndicats seront régies par la loi » (alinéa 3e). "L'étendue, les exceptions et limitations des droits des fonctionnaires publics dans ce domaine seront régies par la loi en tenant compte de la nature de leur service » (alinéa 4e). "Les statuts, direction et fonctionnement des syndicats et des organisations auxquelles ils sont affiliés ne peuvent être contraires aux caractères fondamentaux de la République et aux principes démocratiques » (alinéa $5^{\mathrm{e}}$ ).

Ajoutons que le droit de négociation collective, tout comme le droit de grève, sont également garantis par la Constitution respectivement aux articles 53 et 54 .

Dans le domaine de la fonction publique, il convient de noter que les droits de se syndiquer et de négocier des conventions collectives sont bien reconnus et réglementés. Néanmoins, la grève ne fait pas l'objet d'une protection constitutionnelle et demeure interdite par la loi sur les fonctionnaires publics (Devlet Memurları Kanunu, art. 27), et sanctionnée par le Code pénal Turc (Türk Ceza Kanunu, art. 260).

\section{B - LA RÉGLEMENTATION DES GARANTIES}

La reconnaissance des droits et libertés ne suffisant pas en elle-même, il était indispensable d'instituer des garanties juridiques qui en permettent un exercice effectif. Aussi, le législateur turc s'est-il efforcé d'apporter ces garanties par des dispositions relativement détaillées, tant du point de vue pénal (1) que civil (2).

\section{1 - Les sanctions pénales}

\section{a - La protection pénale contre les entraves aux droits syndicaux}

Intitulé "Entrave à l'usage des droits syndicaux " (sendikal hakların engellenmesi), l'article 118 du Code pénal dispose que : «1) Quiconque fait usage de la violence ou de menaces en vue de forcer quelqu'un à adhérer ou non à un syndicat, à participer ou non à des activités syndicales, à quitter un poste de dirigeant syndical, sera puni d'une peine de prison de six mois à deux ans. 2) En cas d'entrave aux activités d'un syndicat par usage de violence, menaces ou tout autre comportement illégal, la peine prononcée sera entre un et trois ans d'emprisonnement $»$.

Ces dispositions ne sont pas restées lettre morte, et des exemples intéressants illustrent des cas d'entrave qui ont abouti à des sanctions pénales.

En premier lieu, la " victime » pourra être le travailleur et également le syndicat, de sorte que l'action en justice du syndicat intéressé lui-même est recevable ${ }^{2}$. Selon la

$218^{e}$ Chambre pénale de la Cour de Cassation, 5.11.2015, $n^{\circ} 16631 / 10632$, Legal iSGHD, $n^{\circ} 55$, p. 1599. 
jurisprudence établie, des propos tenus par l'employeur ou par un responsable de direction qui menaceraient de "donner des tâches lourdes », de «mettre à la porte » ceux qui sont affiliés à tel syndicat, suffisent à constituer le délit défini à l'article 118 du Code pénal ${ }^{3}$. Même si le salarié ne plie pas devant ces injonctions et garde son affiliation, l'existence de la menace suffit en elle-même à constituer le délit. Tel qu'exprimé par la Chambre pénale de la Cour de Cassation, " vu l'importance que présente la liberté syndicale dans une société démocratique ", toutes les preuves doivent être recueillies, examinées et prises en compte. En l'espèce, l'existence d'actions en justice en vue de réintégrer des salariés licenciés qui ont obtenu gain de cause a été prise en compte. Furent également pris en considération des agissements d'un responsable de direction qui avait dressé une liste d'adhérents pour la communiquer à l'employeur ${ }^{4}$. En ces arrêts, la 18e Chambre pénale fait largement référence à la jurisprudence de la Cour Européenne des Droits de l'Homme, et en particulier aux affaires Karaçay/Turquie (27.03.2007, Requête $n^{\circ}$ 6615/03) et Kaya et Seyhan/Turquie (15.09.2009, Requête n³0946/04).

\section{b - Les amendes administratives}

La Loi n 6356 sur les syndicats et les conventions collectives de travail (Sendikalar ve Toplu iş Sözleşmesi Kanunu) prévoit pour les cas où une peine plus lourde n'est pas encourue, une amende administrative en cas d'enfreinte à l'article 17 sur liberté de s'affilier au syndicat (art. 78/1 c).

\section{2- Les garanties civiles}

La Loi $n^{\circ} 6356$ sur les syndicats et les conventions collectives de travail visent à protéger tout travailleur syndiqué ou non, y compris ceux qui ont participé à des activités syndicales (art. 25). Le droit syndical est assorti de dispositions qui tendent à protéger effectivement le travailleur contre des discriminations lors de l'embauche, pendant la durée du travail et comme cause de licenciement. La protection ainsi prévue concerne différentes étapes.

\section{a - Protection lors de l'embauche et durant la relation de travail}

L'embauche ne saurait être assujettie à l'adhésion ou non à tel ou tel syndicat, et toute clause conventionnelle contraire (dans le contrat de travail ou la convention collective) serait nulle (art. 25/8 de la Loi sur les Syndicats et la convention collective de travail). Face à de telles pratiques, le travailleur aura droit à une indemnité spéciale couramment intitulée « indemnité syndicale » (sendikal tazminat) dont le montant minimum correspond à 1 an de salaires. En l'occurrence, si aucun contrat n'a pu être conclu, l'indemnité devrait être calculée en prenant comme base la rémunération d'un salarié ayant des compétences et situation comparables. Il apparaît ici que la discrimination sera particulièrement difficile à prouver. En effet, à moins que l'employeur n'ait affiché sa volonté de ne recruter par exemple que des travailleurs non syndiqués, comment établir que le choix des recrutements est bien déterminé par une appartenance syndicale ? C'est pourquoi, il est très difficile de trouver des exemples jurisprudentiels où sont invoquées des pratiques antisyndicales lors de l'embauche.

$32^{e}$ Tribunal Pénal de Kütahya, 15.05.2007, n 366/268, Çalışma ve Toplum 2009/1, p. 192 ; $4^{\mathrm{e}}$ Chambre pénale de la Cour de Cassation, 14.07.2010, n⿳ 15172/13889.

$4 \quad 18^{e}$ Chambre pénale de la Cour de Cassation, 7.11.2017, n² 2167/17635, Kazanci. 
Ensuite, après l'embauche, pendant la relation de travail, aucun traitement discriminatoire n'est autorisé par la loi (art. 25/2). Toutefois le principe comporte une exception due au système légal en vigueur qui réserve le bénéfice des avantages pécuniaires des conventions collectives aux seuls travailleurs affiliés au syndicat signataire. Ainsi une différence de traitement est nécessairement admise en ce domaine. Les travailleurs préférant ne pas adhérer au syndicat signataire auront par ailleurs la possibilité de bénéficier des dispositions financières de la convention collective, à condition de verser au syndicat signataire une contribution dite " de solidarité » (dayanışma aidatı). La preuve de la discrimination est à la charge du salarié. Toutefois la preuve se trouve facilitée, puisque «si le travailleur met en avant des faits qui mettent en évidence l'existence de discriminations, l'employeur devra ensuite établir les raisons de son comportement » (art. 25/7). La disposition est inspirée des Directives 2000/435, 2000/786 et 2006/547 de I'Union Européenne où la formulation concernant la preuve est semblable dans les trois textes.

Citons pour exemple l'article 10 relatif à la charge de la preuve de la Directive 2000/78/CE : «1) Les États membres prennent les mesures nécessaires, conformément à leur système judiciaire, afin que, dès lors qu'une personne s'estime lésée par le nonrespect à son égard du principe de l'égalité de traitement et établit, devant une juridiction ou une autre instance compétente, des faits qui permettent de présumer l'existence d'une discrimination directe ou indirecte, il incombe à la partie défenderesse de prouver qu'il n'y a pas eu violation du principe de l'égalité de traitement. 2) Le paragraphe 1 ne fait pas obstacle à l'adoption par les États membres de règles de la preuve plus favorables aux plaignants. 3) Le paragraphe 1 ne s'applique pas aux procédures pénales ».

Nombreux sont les exemples jurisprudentiels de licenciements qui sont intervenus dans un contexte particulièrement révélateur, mettant en évidence la volonté de la direction de pénaliser les travailleurs affiliés à un syndicat, voire même d'éradiquer la présence de syndicats dans l'entreprise.

\section{b - Les protections contre le licenciement}

C'est lorsque le travailleur perd son emploi qu'il saisira le juge, en invoquant l'illicéité de son licenciement. Le salarié allèguera alors que son licenciement était fondé sur une appartenance et/ou une activité syndicale. L'article 25/5 prévoit une protection renforcée, dans la mesure où le travailleur aura la possibilité de demander la réintégration sur son lieu de travail. Pour cela, il devra s'adresser aux tribunaux de travail compétents. Pour ce type d'actions en réintégration seront applicables les dispositions des articles 20 et 21 du Code du travail qui réglementent la protection contre les ruptures du contrat de travail. Dans le cas étudié ici, l'avancée en matière de protection est significative car, en droit commun, seuls sont protégés ceux qui travaillent dans les établissements de plus de 30 salariés et

5 Directive 2000/43/CE du Conseil du 29 juin 2000 relative à la mise en œuvre du principe de l'égalité de traitement entre les personnes sans distinction de race ou d'origine ethnique, Article 8 (Charge de la preuve).

6 Directive 2000/78/CE du Conseil du 27 novembre 2000 portant création d'un cadre général en faveur de l'égalité de traitement en matière d'emploi et de travail, Article 10.

7 Directive 2006/54/CE du Parlement européen et du Conseil du 5 juillet 2006 relative à la mise en $œ u v r e$ du principe de l'égalité des chances et de l'égalité de traitement entre hommes et femmes en matière d'emploi et de travail, Article 19. 
uniquement après 6 mois de service. Or, ces conditions ne sont pas requises en cas de licenciements pour cause syndicale. L'employeur devra alors prouver que le licenciement a été nécessité par la situation de l'entreprise, l'inaptitude ou les agissements du salarié. Si à l'issue du procès le travailleur obtient gain de cause, l'employeur aura la possibilité de choisir soit de réintégrer le salarié, soit de lui verser «l'indemnité syndicale ». Au travailleur requérant est également donné le choix entre agir en vue de sa réintégration, ou exiger le versement de l'indemnité syndicale. En pratique la seconde alternative est plus réaliste, car il est extrêmement rare dans le secteur privé que l'employeur consente à réemployer un salarié qu'il vient de licencier, d'autant plus après une procédure judiciaire.

Cette brève description du système de protection démontre à la fois l'effort du législateur d'apporter une garantie et révèle en même temps le souci d'équilibrer la protection des travailleurs avec les intérêts de l'entreprise. La construction juridique semble suffisamment solide ; mais, dans la pratique, la protection se heurte à plusieurs obstacles.

\section{II - LA DIFFICILE MISE EN ๔UVRE DES GARANTIES JURIDIQUES}

Les difficultés tiennent principalement à la charge de la preuve $(\mathbf{A})$ et à la durée de la procédure (B).

\section{A - Les DifFicultés RELATIVES À LA PREUVE}

La législation présentée ci-dessus facilite dans une certaine mesure l'administration de la preuve lorsqu'il s'agit d'alléguer une discrimination ou une rupture pour cause syndicale. La discrimination au cours de la relation salariale sera prouvée si le salarié " établit des faits de nature à indiquer fortement l'existence d'une discrimination pour cause syndicale » (art. 25/7). En cas de rupture du contrat - le cas le plus fréquent - la charge première incombe à l'employeur, tenu de prouver la cause du licenciement, puis ensuite au travailleur qui devra prouver que la rupture a bien été motivée par une cause syndicale (art. 25/6).

La Cour de Cassation exige la présentation documentée d'un enchaînement de faits indiquant avec suffisamment de crédibilité l'existence du motif syndical. Parmi les faits et documents produits, doivent figurer notamment le nombre de salariés de l'établissement, le nombre de ceux affiliés à un syndicat, avec les dates d'adhésion et de démission, l'existence d'une procédure de négociation collective, et si de nouveaux travailleurs - syndiqués ou non - ont été embauchés lors de ce processus.

Quelques exemples récents de faits et circonstances pris en compte par les juridictions peuvent illustrer la pratique.

Dans un cas, suite à des licenciements pour "motifs économiques », il fut constaté que lors de démarches d'un syndicat pour obtenir l'habilitation à des négociations collectives, l'employeur avait réuni ses salariés et déclaré que "s'ils ne quittent pas le syndicat, l'établissement fermerait ». L'employeur aurait ensuite transporté les salariés au (seul) centre postal ouvert à cette heure, qui se trouve à l'aéroport, afin de leur « permettre de changer d'affiliation syndicale ». Ainsi 258 salariés quittèrent le syndicat; les 36 salariés ayant refusé furent licenciés. En l'occurrence, la Cour de Cassation a estimé avec raison que les agissements de l'employeur étaient contraires à la liberté d'adhérer au syndicat de son choix et a statué en faveur de la réintégration du travailleur requérant et, à défaut, au 
versement de "l'indemnité syndicale » équivalente à un an de salaires ${ }^{8}$. Un autre exemple, plus banal, est celui du salarié auquel la direction reproche des comportements négligents, qui se trouve licencié dans la semaine suivant son adhésion à un syndicat. En l'espèce, la Cour a également tenu compte de l'existence d'une plainte pour entrave à la liberté syndicale dont la procédure pénale suit son cours, renforcée par divers témoignages faisant état de pressions exercées après l'adhésion du requérant au syndicat. Ainsi, la Cour statua pour la réintégration du salarié et/ou le versement de l'indemnité syndicale d'un an de salaires ${ }^{9}$. Enfin, dans un autre cas, de nombreux témoignages faisaient état de pressions du patronat qui procédait notamment à des convocations au bureau du directeur, ce dernier déclarant «ne pas vouloir de syndicat dans son établissement » et enjoignant les salariés à quitter leur syndicat. Par la suite, tous quittèrent le syndicat, à l'exception du requérant qui se trouva licencié dans la semaine suivant son adhésion. En l'occurrence, aucun doute n'était permis sur le motif syndical du licenciement ${ }^{10}$.

Toutefois, il importe de souligner qu'en dehors des affaires où les faits parlent d'euxmêmes pour révéler avec évidence le motif syndical, les témoignages ne suffiront point à établir à eux seuls l'existence d'une violation de la liberté syndicale ${ }^{11}$. Ainsi, un employeur avisé ne pourrait-il pas éventuellement masquer un licenciement syndical, de manière à étaler dans le temps et opérer les ruptures du contrat en dehors d'un contexte de négociation collective?

\section{B - LA DURÉE DE LA PROCÉDURE ET LES SANCTIONS IMPARFAITES}

\section{1 - La durée de la procédure et la phase obligatoire de médiation}

La procédure civile en Turquie est désormais marquée par l'introduction de la voie de l'appel ${ }^{12}$, qui n'existait pas auparavant et contribue aujourd'hui à un allongement supplémentaire de la durée des procès ${ }^{13}$. Pour contrebalancer ce problème et également remédier à la charge de travail des juridictions du travail, la loi prévoit que les procès en réintégration de travailleurs licenciés seraient examinés " en toute urgence » et sans possibilité d'un pourvoi en cassation, les arrêts des Cours d'appel étant définitifs en cas d'actions en réintégration (fondées sur l'art. 20 du Code du travail) ${ }^{14}$. Toutefois, le problème

$89^{e}$ Chambre civile de la Cour de Cassation, 19.11.2018, n 3525/23682, Çalışma ve Toplum, 2019/2, $n^{\circ} 61$, p. 1410.

9 9e Chambre civile de la Cour de Cassation, 10.01.2019, nº 10818/675, Çalışma ve Toplum, 2019/3, $n^{\circ} 62$, p. 2190.

10 22e Chambre civile de la Cour de Cassation, 22.01.2019, n 19282/1614, Çalışma ve Toplum, 2019/3, n 62, p. 2322.

11 Voir par exemple 9e Chambre civile de la Cour de Cassation, 30.09.1996, nº 6756/18050, Çimento Issveren, 1996, p. 29 ; 9e Chambre civile de la Cour de Cassation, 5.10.2004, n 13246/21982, Yargıtay Kararları Dergisi, Août 2005, p. 1226 ; 9 e Chambre civile de la Cour de Cassation, 9.02.2009, $n^{\circ}$ 11004/1854, Çil, Ş. Yargıtay illke Kararları, p. 497.

12 Suite à la Loi n 5235 du 2/03/2005, les « tribunaux civils régionaux » (Bölge adliye mahkemeleri) ont été institués, puis mis en place à partir du $1^{\text {er }}$ septembre 2016.

13 Voir M. Sur, «Turquie", Actualités Juridiques Internationales, Revue de Droit comparé du travail et de la sécurité sociale, 2017/1, p. 204.

14 Voir M. Sur, "Turquie », Actualités juridiques internationales, Revue de Droit comparé du travail et de la sécurité sociale, 2018/1, p. 192. 


\section{LE DROIT SYNDICAL EN TUROUIE}

de la longueur des procès demeure et il faut généralement attendre entre un an et un an et demi pour obtenir un résultat. Or, pendant ce laps de temps, les licenciements auront produit leur effet « dissuasif » dans le milieu du travail et, s'agissant de l'exemplarité de la sanction, il est évident que lorsqu'une sanction intervient tardivement, elle perd de son efficacité.

Une autre réforme de procédure intervenue en $2017^{15}$ a consisté en l'introduction de la « médiation » comme phase obligatoire avant l'ouverture des procès dans le domaine de la relation de travail, que ce soit pour des créances ou pour la réintégration du salarié (art. 3 de la Loi sur les Tribunaux du travail et art. 20 du Code du travail régissant l'action en réintégration du travailleur licencié).

L'introduction de modes alternatifs paraît de prime abord positive, et répond à la Recommandation du Comité des Ministres du Conseil de l'Europe R(86)12 du 16 septembre 1986, qui préconise notamment d'encourager " dans les cas appropriés, le règlement amiable des différends, soit en dehors de l'ordre judiciaire, soit avant ou pendant la procédure judiciaire ». Toutefois, il est fait état de pratiques discutables selon lesquelles des travailleurs en situation de précarité donneraient rapidement leur accord afin de recouvrer sans tarder au moins une partie de leurs créances. La doctrine prédominante en Turquie (dans le champ du droit social) est plutôt hostile au système actuel d'une médiation conçue comme phase obligatoire dans les litiges du travail, et il est inquiétant de voir ainsi contournées des dispositions légales impératives qui visent à protéger le travailleur ${ }^{16}$.

\section{2- Des sanctions peu efficaces}

À l'issue de l'action judiciaire, si le salarié parvient à établir le motif syndical et obtient gain de cause, l'employeur aura le choix entre réembaucher le travailleur, ou lui verser (en plus de l'indemnité de rupture liée à l'ancienneté et l'indemnité de préavis) l'indemnité spéciale dite «syndicale » dont le montant légal minimum correspond à 1 an de salaires. Cette indemnité forfaitaire ne dépend pas de l'existence d'un préjudice et constitue donc une sorte de sanction civile. Dans la pratique, les instances judiciaires ont tendance à statuer au montant minimum, et dans les cas où le tribunal allouerait un montant qui dépasse le minimum légal la Cour de Cassation exige d'en justifier les raisons ${ }^{17}$. La $7^{\text {e }}$ Chambre civile de la Cour de Cassation - fermée par la suite - s'était inspirée de l'article 53 du Code du travail qui règlemente le calcul des congés payés annuels, en échelonnant les majorations effectuées au-dessus de la somme minimale selon la durée de service du salarié ${ }^{18}$.

15 Loi n 7036 du 12.10.2017 publiée au Journal Officiel du 25.10.2017, n³0221.

16 Voir M. Sur, «Turquie », Actualités juridiques internationales, Revue de Droit comparé du travail et de la sécurité sociale, notes 8 et 10, 2018/1, p. 194.

17 ge Chambre civile de la Cour de Cassation, 22.05.2006, n 10439/15150, Çalışma ve Toplum, 2006/3, p. 137.

$187^{\text {e }}$ Chambre civile de la Cour de Cassation, 25.03.2015, n 9209/5525, Çalışma ve Toplum, 2015/3, n 46, p. 225. Ainsi, entre 6 mois et 5 ans d'ancienneté, 1 an de salaires; entre 5 et 15 ans d'ancienneté, 1 mois supplémentaire de salaire ; pour ceux ayant plus de 15 ans d'ancienneté, 2 mois en plus du montant minimum de 1 an de salaires. 
Le recours à cette base de calcul que la législation avait prévue dans un domaine très différent (celui des congés payés annuels), a été critiqué ${ }^{19}$. Toutefois, le critère utilisé avait l'avantage d'une clarté et d'une prévisibilité nécessaires dans le domaine des sanctions ${ }^{20}$. Néanmoins, il semblerait plus pertinent que l'indemnité puisse être calculée en tenant compte non seulement de l'ancienneté du salarié, mais aussi de la gravité de l'entrave à la liberté syndicale, entrave qui affecte à la fois les intérêts particuliers mais aussi le système des relations industrielles et l'ordre public.

Telle fut en quelque sorte la conclusion de la Cour Européenne des Droits de l'Homme, dans son arrêt du 4 avril 2017 (Affaire Tek Gıda Iss Sendikası c. Turquie, Requête n 35009/05)21. En l'espèce, une entreprise d'industrie agroalimentaire avait effectué des licenciements dont le résultat final fut une éradication totale du syndicalisme dans ses établissements. C'est lors du processus d'habilitation à la négociation collective que les salariés avaient été invités par la société à mettre fin à leur adhésion syndicale, sous peine d'être licenciés. Certains obtempérèrent, alors que 40 d'entre eux refusèrent et furent licenciés pour " raisons économiques (fluctuations du marché) » ou pour « insuffisances professionnelles (insuffisance de résultats) ». Saisis en mars 2004, les tribunaux du travail $n^{\circ} 1,2,3,4$ et 5 d'izmir donnèrent gain de cause aux salariés licenciés par des jugements rendus entre juillet et décembre 2004. Les tribunaux ont estimé que les salariés avaient bien été licenciés en raison de leur appartenance syndicale, considérant que la société employeuse (Tukaş) n'avait pas prouvé l'existence de raisons économiques ou d'insuffisances professionnelles justifiant les licenciements litigieux, et ils ordonnèrent la réintégration des personnes licenciées ou, à défaut, le versement à chacun d'une indemnité pour licenciement abusif (syndical) d'un montant correspondant à un an de salaire. Par la suite, la Cour de Cassation confirma ces jugements par des arrêts rendus entre décembre 2004 et juin 2005. La société ne réintégra aucune des personnes licenciées et leur versa l'indemnité ordonnée par les tribunaux. En 2005, le syndicat requérant ne comptait plus aucun adhérent au sein de ces établissements.

La Cour Européenne des Droits de l'Homme, saisie par le syndicat Tek Gıda iş Sendikası le 16 septembre 2005, a rendu son arrêt le 4 avril 2017. La deuxième section (ci-après dénommée «la Cour ») rappelle la jurisprudence selon laquelle si l'article 11 de la Convention a pour objectif essentiel de protéger l'individu contre les ingérences arbitraires des pouvoirs publics dans l'exercice des droits qu'il consacre, il peut en outre impliquer l'obligation positive d'en assurer la jouissance effective (Demir et Baykara, paragraphes 109 et 110, CEDH 2008).

En l'espèce, la Cour estime que les licenciements ont eu deux effets sur les activités du syndicat (paragraphe 60) : "Premièrement, le fait que les décisions judiciaires n'ont pas abouti à la réintégration des salariés licenciés ou, alternativement, à la condamnation de l'entreprise au versement d'indemnités pour licenciements abusifs effectivement dissuasives a eu pour conséquence la perte par le syndicat requérant de ses adhérents

19 S. Süzek, Iş Hukuku, 18 éd, Istanbul, 2019, p. 631 ; H. Sümer, Yargıtay'ın Iş Hukuku ve Sosyal Güvenlik Hukuku Kararlarının Değerlendirilmesi, 2016, Toplu İş ilişkileri, p. 585.

20 Dans ce sens, voir notamment F. Şahlanan, "Sendikal Nedenle Feshe Karşı Açılan İşe İade Davası ve Sendikal Tazminat ", Commentaire de l'arrêt de la 7e Ch. Civile, 25.03.2015, n 9204/5520, iş Hukuku ile Illgili Yargıtay Kararları : Karar Incelemeleri (Ocak 2011-Mart 2016), Tekstil Işveren, p. 341 ; Ö. Ekmekçi, Toplu Iş̧ Hukuku Dersleri, İstanbul, 2018, p. 114.

21 Deuxième Section, arrêt devenu définitif le 4.07.2017. 
au sein de la société Tukaş. Deuxièmement, la non-réintégration des salariés licenciés et l'insuffisance des indemnités accordées par les juridictions aux salariés abusivement licenciés ont eu un effet décourageant sur les autres salariés quant à leur adhésion au syndicat requérant. Ce dernier a ainsi perdu ses chances de garder ses membres ou d'en attirer de nouveaux et d'atteindre le seuil de représentativité indispensable pour négocier des accords collectifs dans la société en question. On ne peut raisonnablement considérer que le préjudice causé par la mise au chômage des salariés syndiqués par la voie d'un licenciement abusif ait été complètement réparé et qu'il ait perdu son effet dissuasif par le seul fait que les personnes licenciées ont perçu une indemnité d'un montant correspondant à un an de salaire, et ce à l'issue d'une procédure judiciaire dont la durée a varié d'un an à un an et demi au lieu de ne pas dépasser les trois mois prévus par la loi. À la lumière de ces éléments, la Cour considère qu'il y a eu une ingérence dans l'exercice par le syndicat requérant, en tant qu'entité distincte de ses membres, de son droit à mener des activités syndicales et des négociations collectives $»$.

La Cour note avec une certaine force au paragraphe 56 que le refus de l'employeur de réintégrer les salariés licenciés et l'octroi d'indemnités insuffisantes pour dissuader l'employeur de procéder à des licenciements abusifs n'enfreignaient pas la loi, telle qu'elle a été interprétée par les décisions judiciaires intervenues en l'espèce. Elle en déduit que la loi y relative, telle qu'appliquée par les tribunaux, n'imposait pas de sanctions suffisamment dissuasives pour l'employeur qui, en procédant à des licenciements massifs abusifs, a réduit à néant la liberté du syndicat requérant de tenter de convaincre des salariés de s'affilier. Par conséquent, ni le législateur ni les juridictions intervenues en l'espèce n'ont rempli leur obligation positive d'assurer au syndicat requérant la jouissance effective de son droit visant à persuader l'employeur d'écouter ce qu'il a à dire au nom de ses membres et, en principe, de son droit de mener des négociations collectives avec lui. Il s'ensuit que le juste équilibre entre, d'une part les intérêts concurrents du syndicat requérant et, d'autre part, de la société dans son ensemble, n'a pas été respecté dans la présente affaire. Dès lors, il y a eu violation de l'article 11 de la Convention sur ce point ${ }^{22}$.

Il convient enfin de relever que la Cour Constitutionnelle turque elle-même a statué dans le même sens dans un autre cas, à l'occasion d'un recours individuel (Recours Anıl Pınar et Ömer Bilge) ${ }^{23}$.

\section{3- Quelques pistes pour des solutions}

Comment assurer une protection efficace ? Les critiques dont fait l'objet la garantie juridique de la liberté syndicale telle qu'elle existe en Turquie sont fondées sur le caractère insuffisamment dissuasif de la sanction ${ }^{24}$, aggravé par une procédure judiciaire trop longue. Or du point de vue de la sanction, il s'avère qu'en calculant le montant brut des salaires annuels, le total s'élève à une somme importante. Ceci démontre que dans certaines

22 Par ailleurs, la durée du procès auprès de la Cour Européenne des Droits de l'Homme apparaît également fort longue (12 années). Le mal était fait, et l'État turc dut payer au syndicat requérant une indemnité pour le « dommage moral » subi (10.000 Euros).

23 Arrêt de la Cour Constitutionnelle, 1 ère Section, 5.07.2017, Journal Officiel du 15.11.2017, n 30241.

24 Voir et comparer dans A. Allamprese et R. Dalmasso, "Les sanctions du licenciement illégitime en France et en Italie : des droits sécurisés ou incitatifs aux licenciements? ", Revue de Droit comparé du travail et de la sécurité sociale, 2019/1, p. 136. 
entreprises la direction cherche à tout prix à éradiquer la présence d'un syndicat dans son entreprise, quel qu'en soit le coût.

Par ailleurs, il existe une catégorie de salariés spécialement protégés (représentants syndicaux et dirigeants syndicaux) pour lesquels la loi prévoit une sanction plus effective (art. 24 de la Loi sur les Syndicats et les conventions collectives de travail)25. Pour licencier un représentant syndical, la loi exige une cause sérieuse de rupture, clairement indiquée par écrit et devant intervenir dans un délai de 6 jours à partir des faits reprochés. Sinon, à l'issue d'un recours auprès des tribunaux du travail, la réintégration du représentant sera prononcée accompagnée du paiement des droits liés au contrat de travail pendant toute la durée de la procédure et jusqu'à réintégration effective. Cette période s'étendra jusqu'à la fin du mandat de représentant, qui est renouvelable. Ainsi, l'employeur qui refuserait de réembaucher serait tenu de payer pour une durée qui peut être très longue tous les droits liés au contrat de travail. Le représentant syndical est par ailleurs également protégé contre des mutations du lieu de travail et des modifications des conditions de travail, soumises à son consentement écrit, faute de quoi la mutation serait nulle. Toutefois, même cette protection comporte une faiblesse en ce qu'elle cesse dès la fin du mandat de représentant ; par la suite, le salarié ne bénéficiera que de la protection de droit commun de l'article 25, ci-avant examinée, avec toutes les limites qu'elle comporte.

Une sanction relativement dissuasive et en même temps plus réaliste et mieux appropriée - en dehors de ce qui est prévu pour les salariés spécialement protégés consisterait, tout en maintenant l'option pour l'employeur de ne pas réintégrer, d'obtenir le jugement définitif dans un court délai et une indemnité dont le montant serait calculé en tenant compte de l'ancienneté du salarié et surtout de la gravité de la violation du droit syndical, des pertes subies par le travailleur, et du potentiel financier de l'entreprise ${ }^{26}$.

Enfin, certains auteurs proposent d'envisager une solution plus radicale qui consisterait en une nullité pure et simple du licenciement, accompagnée du versement des salaires jusqu'à la réintégration effective du salarié, à l'instar de ce qui est prévu pour les représentants syndicaux spécialement protégés à l'article $24^{27}$.

\section{Conclusion}

Le taux de syndicalisation chez les salariés en Turquie est bas et demeure en 2019 à $13,76 \%$, avec un nombre total de 1.894 .170 salariés syndiqués ${ }^{28}$. Or, dans la fonction publique où la liberté syndicale est apparue plus tardivement ${ }^{29}$, la syndicalisation se trouve nettement plus dominante $(66,79 \%$, soit 1.702 .644 fonctionnaires syndiqués). Ainsi, il

25 Pour une analyse des solutions possibles, voir notamment M. Astarlı, « Avrupa Insan Hakları Mahkemesinin Sendika Hakkının Etkili Korunmasına İlişkin Tek Gıda İş Sendikası/Türkiye Kararı ve Türk Hukuku Bakımından Çıkarımlar », Çalışma ve Toplum, 2019/2, n 61, p. 1237.

26 S. Süzek, op. cit., p. 631; M. Sur, Iş Hukuku Toplu Iliş̧kiler, 8ème éd, Ankara, 2019, p. 65 ; M. Astarlı, op. cit., p. 1246.

27 M. Astarlı, op. cit., p. 1253.

28 Statistiques publiées au Journal Officiel du 31.07.2019, n 30848.

29 C'est en 2001 qu'a été promulguée la loi n 4688 sur les syndicats des fonctionnaires - Kamu Görevlileri Sendikaları Kanunu. La loi fut ultérieurement modifiée avec sa nouvelle dénomination de "Loi sur les syndicats et les conventions collectives des fonctionnaires » (Kamu Görevlileri Sendikaları ve Toplu Sözleşme Kanunu). 


\section{LE DROIT SYNDICAL EN TUROUIE}

apparaît sans doute moins risqué pour un fonctionnaire qui jouit des garanties inhérentes à son statut, d'adhérer à un syndicat. On peut également observer que les syndicats majoritaires dans la fonction publique sont souvent plus modérés dans leurs positions.

La Convention de I'OIT $n^{\circ} 87$ précise en son article 11 que chaque État-partie " s'engage à prendre toutes mesures nécessaires et appropriées en vue d'assurer aux travailleurs et aux employeurs le libre exercice de droit syndical ». Or dans le secteur privé, une appartenance syndicale comporte souvent des risques et même une législation telle que celle de la Turquie, favorisant l'adhésion à des syndicats puissants et comportant des protections et garanties non négligeables, ainsi qu'un système de conventions collectives dont bénéficient seuls les adhérents au syndicat signataire, ne suffit pas à encourager les travailleurs à se syndiquer.

En définitive, à côté de la force de la sanction et du système des relations collectives fondées sur la prédominance de syndicats majoritaires, il importe de créer un environnement social propice au dialogue social, un changement des mentalités chez le patronat comme chez les syndicats qui, tout en s'efforçant de maintenir et de faire avancer les droits et avantages des salariés, révisent leur attitude pour parvenir à un dialogue plus constructif et mettent en avant des revendications réalistes, dans un contexte actuel dominé par une crise économique et un taux élevé de chômage ${ }^{30}$.

30 Le taux de chômage s'élevait à 13,9\% en Juillet 2019 selon les statistiques officielles de la TÜiK (Türkiye İstatistik Kurumu - Institut des Statistiques de la Turquie).

\section{MELDA SUR}

Professeur de droit du travail, Faculté de droit, Université Dokuz-Eylül, Izmir, Turquie

Thèmes de recherche : Droit du travail, Relations collectives de travail, droit international du travail, droit international public.

\section{Publications :}

M. Sur (avec N. Turunç), " Turkey », " International Labor and Employment Laws », Turquie, $5^{e}$ éd., volume IIA, in A.-M. Radolinski (dir.), Bloomberg Law, 2018, Arlington, Virginia, p. 20.

M. Sur, "The Union Density Issue in Turkey: Causes and Effects ", Comparative Labor Law \& Policy Journal, vol. 38, n 1, 2016, p. 45.

M. Sur, "Les limites de la liberté contractuelle en droit du travail turc », Des liens et des droits, Mélanges en I'honneur de Jean-Pierre Laborde, Dalloz, Paris 2015, p. 877. 